CERN-PPE/96-085

25 June 1996

\title{
Transverse Momentum Correlations in Hadronic Z Decays
}

\author{
The ALEPH Collaboration
}

\begin{abstract}
Using data obtained with the ALEPH detector at the $\mathrm{Z}$ resonance, a measure based on transverse momentum is shown to exhibit a correlation between the two halves of a hadronic event which cannot be explained by energy-momentum conservation, flavour conservation, the imposition of an event axis or imperfect event reconstruction. Two possible interpretations based on existing Monte Carlo models are examined: a) ARIADNE, with the correlation forming early in the parton shower and with the transition from partons to hadrons playing only a minor part; b) JETSET, with the correlation forming at the fragmentation stage.

A correlation technique based on a jet cluster analysis is used to make a comparison of the models with the data. It is concluded that both non-perturbative and perturbative effects make important contributions to the observed correlation.
\end{abstract}

To be submitted to Zeitschrift für Physik 


\section{The ALEPH Collaboration}

D. Buskulic, I. De Bonis, D. Decamp, P. Ghez, C. Goy, J.-P. Lees, A. Lucotte, M.-N. Minard, J.-Y. Nief, P. Odier, B. Pietrzyk

Laboratoire de Physique des Particules (LAPP), IN ${ }^{2} P^{3}$-CNRS, 74019 Annecy-le-Vieux Cedex, France

M.P. Casado, M. Chmeissani, J.M. Crespo, M. Delfino, I. Efthymiopoulos ${ }^{1}$ E. Fernandez, M. FernandezBosman, Ll. Garrido, ${ }^{15}$ A. Juste, M. Martinez, S. Orteu, C. Padilla, I.C. Park, A. Pascual, J.A. Perlas, I. Riu, F. Sanchez, F. Teubert

Institut de Fisica d'Altes Energies, Universitat Autonoma de Barcelona, 08193 Bellaterra (Barcelona), $\operatorname{Spain}^{7}$

A. Colaleo, D. Creanza, M. de Palma, G. Gelao, M. Girone, G. Iaselli, G. Maggi, M. Maggi, N. Marinelli, S. Nuzzo, A. Ranieri, G. Raso, F. Ruggieri, G. Selvaggi, L. Silvestris, P. Tempesta, A. Tricomi, ${ }^{3}$ G. Zito

Dipartimento di Fisica, INFN Sezione di Bari, 70126 Bari, Italy

X. Huang, J. Lin, Q. Ouyang, T. Wang, Y. Xie, R. Xu, S. Xue, J. Zhang, L. Zhang, W. Zhao

Institute of High-Energy Physics, Academia Sinica, Beijing, The People's Republic of China ${ }^{8}$

R. Alemany, A.O. Bazarko, G. Bonvicini, ${ }^{23}$ P. Bright-Thomas, M. Cattaneo, P. Comas, P. Coyle, H. Drevermann, R.W. Forty, M. Frank, R. Hagelberg, J. Harvey, P. Janot, B. Jost, E. Kneringer, J. Knobloch, I. Lehraus, G. Lutters, E.B. Martin, P. Mato, A. Minten, R. Miquel, Ll.M. Mir, ${ }^{2}$ L. Moneta, T. Oest, ${ }^{20}$ A. Pacheco, J.-F. Pusztaszeri, F. Ranjard, P. Rensing, ${ }^{12}$ G. Rizzo, L. Rolandi, D. Schlatter, M. Schmelling, ${ }^{24}$ M. Schmitt, O. Schneider, W. Tejessy, I.R. Tomalin, A. Venturi, H. Wachsmuth, A. Wagner

European Laboratory for Particle Physics (CERN), 1211 Geneva 23, Switzerland

Z. Ajaltouni, A. Barrès, C. Boyer, A. Falvard, P. Gay, C . Guicheney, P. Henrard, J. Jousset, B. Michel, S. Monteil, J-C. Montret, D. Pallin, P. Perret, F. Podlyski, J. Proriol, P. Rosnet, J.-M. Rossignol

Laboratoire de Physique Corpusculaire, Université Blaise Pascal, $I N^{2} P^{3}-C N R S$, Clermont-Ferrand, 63177 Aubière, France

T. Fearnley, J.B. Hansen, J.D. Hansen, J.R. Hansen, P.H. Hansen, B.S. Nilsson, B. Rensch, A. Wäänänen

Niels Bohr Institute, 2100 Copenhagen, Denmark ${ }^{9}$

A. Kyriakis, C. Markou, E. Simopoulou, I. Siotis, A. Vayaki, K. Zachariadou

Nuclear Research Center Demokritos (NRCD), Athens, Greece

A. Blondel, G. Bonneaud, J.C. Brient, P. Bourdon, A. Rougé, M. Rumpf, A. Valassi, ${ }^{6}$ M. Verderi, H. Videau ${ }^{21}$

Laboratoire de Physique Nucléaire et des Hautes Energies, Ecole Polytechnique, IN ${ }^{2} P^{3}$-CNRS, 91128 Palaiseau Cedex, France

D.J. Candlin, M.I. Parsons

Department of Physics, University of Edinburgh, Edinburgh EH9 3JZ, United Kingdom ${ }^{10}$

E. Focardi, ${ }^{21}$ G. Parrini

Dipartimento di Fisica, Università di Firenze, INFN Sezione di Firenze, 50125 Firenze, Italy

M. Corden, C. Georgiopoulos, D.E. Jaffe

Supercomputer Computations Research Institute, Florida State University, Tallahassee, FL 32306$4052, U_{S A}{ }^{13,14}$

A. Antonelli, G. Bencivenni, G. Bologna ${ }^{4}$ F. Bossi, P. Campana, G. Capon, D. Casper, V. Chiarella, G. Felici, P. Laurelli, G. Mannocchi ${ }^{5}$ F. Murtas, G.P. Murtas, L. Passalacqua, M. Pepe-Altarelli

Laboratori Nazionali dell'INFN (LNF-INFN), 00044 Frascati, Italy

L. Curtis, S.J. Dorris, A.W. Halley, I.G. Knowles, J.G. Lynch, V. O'Shea, C. Raine, P. Reeves, J.M. Scarr, 
K. Smith, P. Teixeira-Dias, A.S. Thompson, F. Thomson, S. Thorn, R.M. Turnbull

Department of Physics and Astronomy, University of Glasgow, Glasgow G12 8QQ,United Kingdom ${ }^{10}$

U. Becker, C. Geweniger, G. Graefe, P. Hanke, G. Hansper, V. Hepp, E.E. Kluge, A. Putzer, M. Schmidt, J. Sommer, H. Stenzel, K. Tittel, S. Werner, M. Wunsch

Institut für Hochenergiephysik, Universität Heidelberg, 69120 Heidelberg, Fed. Rep. of Germany ${ }^{16}$

D. Abbaneo, R. Beuselinck, D.M. Binnie, W. Cameron, P.J. Dornan, A. Moutoussi, J. Nash, J.K. Sedgbeer, A.M. Stacey, M.D. Williams

Department of Physics, Imperial College, London SW7 2BZ, United Kingdom ${ }^{10}$

G. Dissertori, P. Girtler, D. Kuhn, G. Rudolph

Institut für Experimentalphysik, Universität Innsbruck, 6020 Innsbruck, Austria ${ }^{18}$

A.P. Betteridge, C.K. Bowdery, P. Colrain, G. Crawford, A.J. Finch, F. Foster, G. Hughes, T. Sloan, M.I. Williams

Department of Physics, University of Lancaster, Lancaster LA1 4YB, United Kingdom ${ }^{10}$

A. Galla, I. Giehl, A.M. Greene, C. Hoffmann, K. Jakobs, K. Kleinknecht, G. Quast, B. Renk, E. Rohne, H.-G. Sander, P. van Gemmeren, C. Zeitnitz

Institut für Physik, Universität Mainz, 55099 Mainz, Fed. Rep. of Germany ${ }^{16}$

J.J. Aubert, ${ }^{21}$ A.M. Bencheikh, C. Benchouk, A. Bonissent, G. Bujosa, D. Calvet, J. Carr, C. Diaconu,

F. Etienne, N. Konstantinidis, P. Payre, D. Rousseau, M. Talby, A. Sadouki, M. Thulasidas, K. Trabelsi

Centre de Physique des Particules, Faculté des Sciences de Luminy, IN ${ }^{2} P^{3}-C N R S, 13288$ Marseille,

France

M. Aleppo, F. Ragusa ${ }^{21}$

Dipartimento di Fisica, Università di Milano e INFN Sezione di Milano, 20133 Milano, Italy

C. Bauer, R. Berlich, W. Blum, V. Büscher, H. Dietl, F. Dydak, ${ }^{21}$ G. Ganis, C. Gotzhein, H. Kroha, G. Lütjens, G. Lutz, W. Männer, H.-G. Moser, R. Richter, A. Rosado-Schlosser, S. Schael, R. Settles, H. Seywerd, R. St. Denis, H. Stenzel, W. Wiedenmann, G. Wolf

Max-Planck-Institut für Physik, Werner-Heisenberg-Institut, 80805 München, Fed. Rep. of Germany ${ }^{16}$

J. Boucrot, O. Callot, Y. Choi, ${ }^{26}$ A. Cordier, M. Davier, L. Duflot, J.-F. Grivaz, Ph. Heusse, A. Höcker, A. Jacholkowska, M. Jacquet, D.W. Kim, ${ }^{19}$ F. Le Diberder, J. Lefrançois, A.-M. Lutz, I. Nikolic, H.J. Park, ${ }^{19}$ M.-H. Schune, S. Simion, J.-J. Veillet, I. Videau, D. Zerwas

Laboratoire de l'Accélérateur Linéaire, Université de Paris-Sud, IN ${ }^{2} P^{3}$-CNRS, 91405 Orsay Cedex, France

P. Azzurri, G. Bagliesi, G. Batignani, S. Bettarini, C. Bozzi, G. Calderini, M. Carpinelli, M.A. Ciocci, V. Ciulli, R. Dell'Orso, R. Fantechi, I. Ferrante, L. Foà, ${ }^{1}$ F. Forti, A. Giassi, M.A. Giorgi, A. Gregorio, F. Ligabue, A. Lusiani, P.S. Marrocchesi, A. Messineo, F. Palla, G. Sanguinetti, A. Sciabà, P. Spagnolo, J. Steinberger, R. Tenchini, G. Tonelli, ${ }^{25}$ C. Vannini, P.G. Verdini, J. Walsh

Dipartimento di Fisica dell'Università, INFN Sezione di Pisa, e Scuola Normale Superiore, 56010 Pisa, Italy

G.A. Blair, L.M. Bryant, F. Cerutti, J.T. Chambers, Y. Gao, M.G. Green, T. Medcalf, P. Perrodo, J.A. Strong, J.H. von Wimmersperg-Toeller

Department of Physics, Royal Holloway \& Bedford New College, University of London, Surrey TW20 OEX, United Kingdom ${ }^{10}$

D.R. Botterill, R.W. Clifft, T.R. Edgecock, S. Haywood, P. Maley, P.R. Norton, J.C. Thompson, A.E. Wright

Particle Physics Dept., Rutherford Appleton Laboratory, Chilton, Didcot, Oxon OX11 OQX, United Kingdom ${ }^{10}$

B. Bloch-Devaux, P. Colas, S. Emery, W. Kozanecki, E. Lançon, M.C. Lemaire, E. Locci, B. Marx, P. Perez, J. Rander, J.-F. Renardy, A. Roussarie, J.-P. Schuller, J. Schwindling, A. Trabelsi, B. Vallage CEA, DAPNIA/Service de Physique des Particules, CE-Saclay, 91191 Gif-sur-Yvette Cedex, France ${ }^{17}$ 
S.N. Black, J.H. Dann, R.P. Johnson, H.Y. Kim, A.M. Litke, M.A. McNeil, G. Taylor

Institute for Particle Physics, University of California at Santa Cruz, Santa Cruz, CA 95064, USA ${ }^{22}$

C.N. Booth, R. Boswell, C.A.J. Brew, S. Cartwright, F. Combley, A. Koksal, M. Letho, W.M. Newton, J. Reeve, L.F. Thompson

Department of Physics, University of Sheffield, Sheffield S3 $7 R H$, United Kingdom ${ }^{10}$

A. Böhrer, S. Brandt, G. Cowan, C. Grupen, J. Minguet-Rodriguez, F. Rivera, P. Saraiva, L. Smolik, F. Stephan,

Fachbereich Physik, Universität Siegen, 57068 Siegen, Fed. Rep. of Germany ${ }^{16}$

M. Apollonio, L. Bosisio, R. Della Marina, G. Giannini, B. Gobbo, G. Musolino

Dipartimento di Fisica, Università di Trieste e INFN Sezione di Trieste, 34127 Trieste, Italy

J. Rothberg, S. Wasserbaech

Experimental Elementary Particle Physics, University of Washington, WA 98195 Seattle, U.S.A.

S.R. Armstrong, P. Elmer, Z. Feng, ${ }^{27}$ D.P.S. Ferguson, Y.S. Gao, ${ }^{28}$ S. González, J. Grahl, T.C. Greening, O.J. Hayes, H. Hu, P.A. McNamara III, J.M. Nachtman, W. Orejudos, Y.B. Pan, Y. Saadi, I.J. Scott, A.M. Walsh, ${ }^{29}$ Sau Lan Wu, X. Wu, J.M. Yamartino, M. Zheng, G. Zobernig

Department of Physics, University of Wisconsin, Madison, WI 53706, USA ${ }^{11}$

\footnotetext{
${ }^{1}$ Now at CERN, 1211 Geneva 23, Switzerland.

${ }^{2}$ Supported by Dirección General de Investigación Científica y Técnica, Spain.

${ }^{3}$ Also at Dipartimento di Fisica, INFN, Sezione di Catania, Catania, Italy.

${ }^{4}$ Also Istituto di Fisica Generale, Università di Torino, Torino, Italy.

${ }^{5}$ Also Istituto di Cosmo-Geofisica del C.N.R., Torino, Italy.

${ }^{6}$ Supported by the Commission of the European Communities, contract ERBCHBICT941234.

${ }^{7}$ Supported by CICYT, Spain.

${ }^{8}$ Supported by the National Science Foundation of China.

${ }^{9}$ Supported by the Danish Natural Science Research Council.

${ }^{10}$ Supported by the UK Particle Physics and Astronomy Research Council.

${ }^{11}$ Supported by the US Department of Energy, grant DE-FG0295-ER40896.

${ }^{12}$ Now at Dragon Systems, Newton, MA 02160, U.S.A.

${ }^{13}$ Supported by the US Department of Energy, contract DE-FG05-92ER40742.

${ }^{14}$ Supported by the US Department of Energy, contract DE-FC05-85ER250000.

${ }^{15}$ Permanent address: Universitat de Barcelona, 08208 Barcelona, Spain.

${ }^{16}$ Supported by the Bundesministerium für Forschung und Technologie, Fed. Rep. of Germany.

${ }^{17}$ Supported by the Direction des Sciences de la Matière, C.E.A.

${ }^{18}$ Supported by Fonds zur Förderung der wissenschaftlichen Forschung, Austria.

${ }^{19}$ Permanent address: Kangnung National University, Kangnung, Korea.

${ }^{20}$ Now at DESY, Hamburg, Germany.

${ }^{21}$ Also at CERN, 1211 Geneva 23, Switzerland.

${ }^{22}$ Supported by the US Department of Energy, grant DE-FG03-92ER40689.

${ }^{23}$ Now at Wayne State University, Detroit, MI 48202, USA.

${ }^{24}$ Now at Max-Plank-Institüt für Kernphysik, Heidelberg, Germany.

${ }^{25}$ Also at Istituto di Matematica e Fisica, Università di Sassari, Sassari, Italy.

${ }^{26}$ Permanent address: Sung Kyun Kwon University, Suwon, Korea.

${ }^{27}$ Now at The Johns Hopkins University, Baltimore, MD 21218, U.S.A.

${ }^{28}$ Now at Harvard University, Cambridge, MA 02138, U.S.A.

${ }^{29}$ Now at Rutgers University, Piscataway, NJ 08855-0849, U.S.A.
} 


\section{Introduction}

Correlation studies of the hadronization process in high energy $e^{+} e^{-}$annihilation can give insight into the underlying QCD processes and also provide a test of hadronization models. Published work so far at LEP energies on event correlations has concentrated on aspects of particle multiplicity and average transverse momentum of charged particles. In addition to their work on multiplicity correlations, DELPHI $[1,2]$ examined the correlation between event multiplicity and average transverse momentum in and out of the event plane. From a classification of events in terms of the number of jets they concluded that hard gluon radiation was the main source of the correlation observed. OPAL [3] looked in particular at charged particle correlations between the two sides of an event (defined by the thrust axis). They also classified events in terms of numbers of jets finding that, while flavour effects were important for the two jet class, the general correlation could be understood as resulting from a superposition of the different $n$-jet classes, again a hard gluon effect.

The present analysis follows a somewhat different path. The thrust axis is determined and the event is partitioned by a plane normal to this axis. At this and subsequent stages all particles, charged and neutral, are included. For each half a new axis is defined by the vector sum of the momenta over all the particles and the quantity

$$
P_{t}=\sum_{\text {half event }}\left|p_{t i}\right|
$$

is formed, where $p_{t i}$ is the transverse momentum of the $i^{\text {th }}$ particle with respect to the halfevent axis. (Given perfect reconstruction of all particles and absence of initial state radiation, the thrust axis and the two half axes would coincide). The quantities $P_{t 1}$ and $P_{t 2}$ are referred to as the transverse momenta of the two half-events. The labels 1 and 2 are chosen at random. A two-jet event will have relatively low $P_{t}$ on both sides; one with three jets will be low on one side and high on the other. Evidence for a significant positive correlation in transverse momentum between the two halves, not hitherto reported, is presented and discussed.

Apart from its generality, the use of $P_{t}$ as a measure of the behaviour of a half event has several advantages. It is insensitive to boosts along the axis (provided no particles change sides) and therefore is not affected by varying energies on the other side; it is collinear safe which, together with the use of the thrust axis, facilitates a key comparison between parton and hadron levels and, unlike jet mass, for example, it has good discrimination even for narrow jets.

\section{Event Selection and Analysis}

The ALEPH detector [4] and its performance [5] are described in detail elsewhere. The analysis is based on data acquired in 1992 at the $\mathrm{Z}$ resonance. Candidate hadron events are required to have at least five charged tracks with polar angle $\theta$ to the beam axis such that: $|\cos \theta|<0.95$; the distance of closest approach to the beam axis is $<2 \mathrm{~cm}$; the distance from the vertex to the interaction point along the beam axis is $<10 \mathrm{~cm}$ and there are at least four hits in the principal 
tracking chamber, the Time Projection Chamber. The total visible energy of all such tracks measured must exceed $10 \%$ of the total centre of mass energy. Approximately 700k hadron events survive these cuts.

Charged and neutral particles are reconstructed as 'energy flow objects' [5]. The thrust axis is required to lie in the range of polar angles from 35 to $145^{\circ}$ with respect to the beam direction; events with total energy $<70 \mathrm{GeV}$ are rejected and any remaining $\tau^{+} \tau^{-}$pairs are removed by requiring that at least one side has $P_{t}>2 \mathrm{GeV} / \mathrm{c}$.

Figure 1 summarizes the key features of the ALEPH raw data. The comparison with JETSET will be discussed in section 3. Figure 1(a) is a scatter plot of $P_{t 1} \mathrm{v} P_{t 2}$. The $\tau$ pairs have been included in this plot. The points are largely confined to the triangle bounded by the line from $(0,60)$ to $(60,0)$. This boundary is mainly an effect of the thrust axis. There is a high concentration of events along broad bands centred on $6 \mathrm{GeV} / \mathrm{c}$. The average measured $P_{t}$ for all events is about $10 \mathrm{GeV} / \mathrm{c}$. For convenience an event for which both sides have $P_{t}$ less than this average will be referred to as being in the 'two jet' region. The $P_{t}$ distribution is shown in Fig. 1(b). For reasons explained below, this distribution refers to events in which $P_{t}<25 \mathrm{GeV} / \mathrm{c}$ on both sides.

The correlation coefficient between pairs of quantities $(x, y)$ is defined as

$$
C(x, y)=\frac{\overline{x y}-\bar{x} \bar{y}}{\sigma_{x} \sigma_{y}}
$$

where $\sigma_{x}$ and $\sigma_{y}$ are the standard deviations of the $x$ and $y$ distributions $\left(\bar{x}=\bar{y}=\overline{P_{t}}\right.$, $\sigma_{x}=\sigma_{y}=\sigma$ ). The correlation between the two sides of an event is now examined for subsets of the events that satisfy $P_{t 1}, P_{t 2}<P_{t, \max }$, Fig.1 (c). With $P_{t, \max }=60 \mathrm{GeV} / \mathrm{c}$, essentially the entire data set, there is a positive correlation of about 0.04. As $P_{t, \max }$ is lowered, $C$ slowly rises to around 0.08 and remains near this value into the two jet region. The correlation for three values of $P_{t, \max }(60,25$ and $10 \mathrm{GeV} / \mathrm{c})$ are given in Table 1 . The error quoted is statistical. The rise in $C$ as $P_{t, \max }$ is reduced in value from $60 \mathrm{GeV} / \mathrm{c}$ is easy to understand in terms of the scatter plot as the cut first removes the events in the high $P_{t}$ corners. The correlation at the plateau value is the subject of the present paper.

The analysis will concentrate on subsets of events defined by $P_{t, \max }=25 \mathrm{GeV} / \mathrm{c}$, a cut chosen to be low enough to be safely inside the triangle seen in the scatter plot but also high enough to include almost $90 \%$ of the data and to make flavour and detector effects relatively small. Furthermore, the density of events in the region of this cut is low, therefore small changes in measured $P_{t}$ have relatively little effect on the sample.

\begin{tabular}{|c|c|c|c|c|}
\hline$P_{t, \text { max }}, \mathrm{GeV} / \mathrm{c}$ & Number of events & $\overline{P_{t}} \mathrm{GeV} / \mathrm{c}$ & $\sigma \mathrm{GeV} / \mathrm{c}$ & $C\left(P_{t 1}, P_{t 2}\right)$ \\
\hline 60 & $513 \mathrm{~K}$ & 10.0 & 7.33 & $\mathbf{0 . 0 4 4} \pm \mathbf{0 . 0 0 1 4}$ \\
25 & $457 \mathrm{~K}$ & 8.69 & 5.01 & $0.084 \pm \mathbf{0 . 0 0 1 5}$ \\
10 & $232 \mathrm{~K}$ & 5.90 & 1.96 & $0.073 \pm \mathbf{0 . 0 0 2 0}$ \\
\hline
\end{tabular}

Table 1. Correlation coefficients for three subsets of the 1992 ALEPH data. 
Figure 1(d) shows how the average value of $P_{t}$ on one side varies with the value selected for the other $\left(P_{t, \text { max }}=25 \mathrm{GeV} / \mathrm{c}\right)$. This function will be written as $\overline{P_{t 1}}\left(P_{t 2}\right)$ but the 1 and 2 are arbitrary and each event contributes twice to the average. It is seen that $\overline{P_{t 1}}$ rises by about 1.3 $\mathrm{GeV} / \mathrm{c}$ until $P_{t 2}$ reaches about $11 \mathrm{GeV} / \mathrm{c}$ above which value the rate of rise is very much reduced. A rise at low $P_{t 2}$ followed by a near plateau above $10 \mathrm{GeV} / \mathrm{c}$ or so is a recurring theme of this investigation. The variation of $\overline{P_{t 1}}$ with $P_{t 2}$ is another manifestation of the correlation between the two sides. While such a plot is of interest, its functional form is strongly influenced by the sharp rise and slower fall typical of a $P_{t}$ distribution, Fig. 1(b). If, for example, a linear coupling $P_{t 1} \longrightarrow P_{t 1}+\epsilon\left(P_{t 2}-P_{t 1}\right) ; P_{t 2} \longrightarrow P_{t 2}+\epsilon\left(P_{t 1}-P_{t 2}\right)$ is introduced between two unassociated half-events, the resulting $\overline{P_{t 1}}\left(P_{t 2}\right)$ has the same general behaviour seen in Fig. 1(d). The main quantitative results will be expressed in terms of values of the correlation at $P_{t, \max }=25 \mathrm{GeV} / \mathrm{c}$.

Correlations can arise in many ways, ranging from detector effects to conservation laws. Certain general comments, however, can be made.

The results are found to be insensitive to an increase in the minimum total energy threshold from 70 to $75 \mathrm{GeV}$ or to a reduction in the polar angular range from $35-145^{\circ}$ to $45-135^{\circ}$. Further subdivision by polar angle confirms that there is no significant contribution to the correlation from the range of polar angles included in the data set.

Forcing an axis on an event can have a large effect, including the introduction of correlations, with complex high $P_{t}$ events (e.g. the boundary triangle in Fig. 1(a)). However the concern here is with a correlation that remains high down to the two jet region. The axis is used only to partition the event, a separation that must be nearly unambiguous at the low $P_{t}$ end.

Reconstruction errors, missing neutrinos and initial state radiation could be important were $P_{t}$ to be measured on each side with respect to a single axis since if, for any reason, the two halves of an event were not back-to-back, the compromise axis would lead to a positive correlation. However, once partitioned, each half is measured with respect to its own axis.

As a consequence of energy-momentum conservation, higher than average $P_{t}$ on one side will normally correspond to higher than average effective mass and therefore lower than average energy on the opposite side. Since the opposite $P_{t}$ also will tend to fall, such a division, if anything, should lead to a negative correlation. Over the range of interest the variation is small; the average measured energy opposite $P_{t}=3 \mathrm{GeV} / \mathrm{c}$ is $45.6 \mathrm{GeV}$, opposite $11 \mathrm{GeV} / \mathrm{c}$ is 44.4 $\mathrm{GeV}$ and opposite $20 \mathrm{GeV} / \mathrm{c}$ has only fallen to $42.4 \mathrm{GeV}$.

The correlation was found not to be the result of anomalous or spurious events in the sample. If the distribution of $P_{t 1}$, as in Fig. 1(b), is examined as a function of $P_{t 2}$ (not shown here), a general shift to higher $P_{t 1}$ values is seen as $P_{t 2}$ is raised from $3 \mathrm{GeV} / \mathrm{c}$ to $11 \mathrm{GeV} / \mathrm{c}$, with little further change up to $P_{t 2}=25 \mathrm{GeV} / \mathrm{c}$.

\section{The JETSET Parton Shower Model}

A large sample of Monte Carlo events were generated using the program JETSET 7.3 [6, 7 ] which has been tuned to the data [8]. These events were passed through a detailed simulation of the detector and the analysis chain. They are referred to as 'reconstructed' and are used in 
several ways: as an aid in the investigation of flavour effects; to give an estimate of the possible effect of the ALEPH detector on the correlation; as a source of hadron and parton events for comparison purposes and finally for a direct comparison with the ALEPH events themselves.

The ALEPH and JETSET $P_{t}$ distributions, Fig. 1(b), are in reasonable agreement. Figure 1(c) shows that the full set of JETSET events, i.e. $P_{t, \max }=60 \mathrm{GeV} / \mathrm{c}$, has a positive correlation which, like the ALEPH data, rises with falling $P_{t, \max }$ but remains significantly below the ALEPH data for all $P_{t, \max }$ values above about $10 \mathrm{GeV} / \mathrm{c}$. Clearly there is some correlating mechanism within JETSET, though it fails to describe the data well when outside the two jet region. Figure 1(d) gives the behaviour of $\overline{P_{t 1}}$ as a function of $P_{t 2}$ : qualitatively JETSET shows the same rise up to $\approx 11 \mathrm{GeV} / \mathrm{c}$ and the near plateau above, though the magnitude of the rise, about 1.0 $\mathrm{GeV} / \mathrm{c}$, is significantly smaller and, unlike the data, there is very little variation in $\overline{P_{t}}$ above 11 $\mathrm{GeV} / \mathrm{c}$.

Quantitative comparisons at $P_{t, \text { max }}=25 \mathrm{GeV} / \mathrm{c}$ can be made from the first two rows of Table 2. Average $P_{t}$ is a little higher, and the $P_{t}$ distribution a little wider, for the JETSET events. However, as noted, the main discrepancy lies in the value of $C$ which is about $30 \%$ low in JETSET. The selection of the subset of reconstructed events for which there is no initial state radiation has only a marginal effect; it raises the value of $C$ to $0.060 \pm .0017$. The errors quoted are statistical only.

\begin{tabular}{|c|c|c|c|c|}
\hline & Accepted events & $\overline{P_{t}} \mathrm{GeV} / \mathrm{c}$ & $\sigma \mathrm{GeV} / \mathrm{c}$ & $C$ \\
\hline ALEPH data & $457 \mathrm{k}$ & 8.69 & 5.01 & $\mathbf{0 . 0 8 4} \pm \mathbf{0 . 0 0 1 5}$ \\
JETSET rec. & $599 \mathrm{k}$ & 8.86 & 5.10 & $\mathbf{0 . 0 5 9} \pm \mathbf{0 . 0 0 1 3}$ \\
JETSET gen. & $606 \mathrm{k}$ & 8.45 & 5.07 & $\mathbf{0 . 0 6 3} \pm \mathbf{0 . 0 0 1 3}$ \\
\hline
\end{tabular}

Table 2. Comparisons of ALEPH data with reconstructed and generated JETSET events. All numbers quoted refer to the subset of events with $P_{t 1}, P_{t 2}<P_{t, \max }=25 \mathrm{GeV} / \mathrm{c}$.

\subsection{Flavour conservation}

The events are a mixture of several flavours. If there were just two flavours, say $d \bar{d}$ and $b \bar{b}$, with a fraction $f$ of $d \bar{d}$ and a difference in average $P_{t}, \overline{P_{t, b}}-\overline{P_{t, d}}=\epsilon$, then assuming that the correlation for either flavour by itself was small and that changes in $\sigma$ with $\epsilon$ could be neglected, the presence of the mixture would increase the correlation by

$$
\delta C=\frac{f(1-f) \epsilon^{2}}{\sigma^{2}}
$$

At $\sigma=5.0 \mathrm{GeV} / \mathrm{c}, \delta C$ has a maximum value $(f=0.5)$ of $0.01 \times \epsilon^{2}$.

When JETSET is used to estimate the detailed variation of $\overline{P_{t}}$ with flavour, the only significant variation is from $b \bar{b}$ events which have a $\Delta \overline{P_{t}} \approx+0.70 \mathrm{GeV} / \mathrm{c}$ with respect to the 
others. Direct evidence from the ALEPH data that flavour effects are small in the reconstructed hadron events is obtained by comparing average $\overline{P_{t}}$ for a sample of events within $P_{t, \text { max }}=25$ $\mathrm{GeV} / \mathrm{c}$ with the $\overline{P_{t}}$ of half events from the same sample opposite a b-tag [9]. A probability of $<0.001$ for a light quark to pass the tagging cut is imposed. A similar comparison is made for reconstructed JETSET events. The difference in $\overline{P_{t}}$ is both small and well described by JETSET (Table 3).

\begin{tabular}{|c|c|c|c|}
\hline & All events & Opposite b-tag & $\overline{P_{t}}$ difference \\
\hline ALEPH & $8.71 \pm .012$ & $\mathbf{9 . 2 0} \pm . \mathbf{0 4 1}$ & $\mathbf{0 . 4 9} \pm .04$ \\
JETSET & $8.82 \pm .008$ & $9.37 \pm .033$ & $\mathbf{0 . 5 5} \pm .03$ \\
\hline
\end{tabular}

Table 3. Average $P_{t}$ values for samples of events with and without a b-tag on the opposite side.

A detailed calculation based on JETSET values for all flavours gives $\delta C=0.003$ for $P_{t, \max }$ $=25 \mathrm{GeV} / \mathrm{c}$. This is small compared to the observed value of $C$ of 0.084 . The sensitivity to a flavour mixture can increase rapidly as $P_{t, \max }$, and therefore $\sigma$, is lowered.

\subsection{Correcting for Detector effects}

JETSET events have been compared at reconstructed and generator level. For generator level, neutrinos were excluded and all particles with mean lifetimes less than 1ns were forced to decay. Figures are given in Table 2 for the comparison at $P_{t, \max }=25 \mathrm{GeV} / \mathrm{c}$; between generation and reconstruction there is a small fall in $C$ from 0.063 to 0.059 .

The information from JETSET events can be used to predict the reconstructed event correlation for other generators for which a full reconstruction simulation is not available. Given a class of events that, as generated, lie in a $1 \mathrm{GeV} / \mathrm{c}$ box around some $\left(P_{t 1}, P_{t 2}\right)$, on reconstruction the same events will give rise to a range of $P_{t 1}, P_{t 2}$ values. The assumption is that this detector transformation is typical of the class. A second generator will, in general, have a different proportion of generated events in the same box. A weight array is formed by the ratio of the two proportions at each $\left(P_{t 1}, P_{t 2}\right)$. The corresponding reconstructed JETSET data set is re-analyzed, each event being given the appropriate weighting to correspond to the new generator and the resulting correlation determined. The method does not allow for direct correction backwards from reconstructed to generator level but, as noted, the correction for $P_{t, \max }=25 \mathrm{GeV} / \mathrm{c}$ is small.

The significant discrepancy between ALEPH data and JETSET, Fig. 1(c), coupled with the narrow $P_{t}$ distribution, Fig. 1(b), makes a reliable correction for the contribution of detector effects to a measured correlation difficult. This problem is exacerbated for subsets of the data at low $P_{t, \text { max }}$. Therefore no attempt at a correction is made at other than the standard $P_{t, \text { max }}$ of $25 \mathrm{GeV} / \mathrm{c}$. One approach is simply to assume that event reconstruction loses a certain fraction of $C$, i.e. 0.004 parts in 0.059 . This gives a corrected ALEPH value at a $P_{t, \max }$ of $25 \mathrm{GeV} / \mathrm{c}$ 
of 0.089. Another, based on the method described above and probably to be preferred, is to increase the JETSET correlation by introducing an additional coupling at generator level:

$$
P_{t 1} \longrightarrow P_{t 1}+\epsilon\left(P_{t 2}-P_{t 1}\right), P_{t 2} \longrightarrow P_{t 2}+\epsilon\left(P_{t 1}-P_{t 2}\right)
$$

to make a new set to which the weighting technique can be applied. The value of $\epsilon$, chosen to give a new reconstructed value close to that observed, is $\epsilon=0.017$. This indicates a correction of +0.007 , making a final corrected value of $C=0.091 \pm 0.004$ where a conservative error of half the correction has been assumed.

\section{Interpretation of the correlation}

The correlation is a feature of the set of events as a whole; it is at its maximum value at a $P_{t, \max }$ cut which includes nearly $90 \%$ of the data. Whatever the source of the correlation, the effect must be distributed over the set of events; its identification probably is only possible by comparing the observations with the results obtained via Monte Carlo generators into which possible hypotheses are incorporated.

The question can be asked 'Do the two halves start correlated or is the correlation acquired during fragmentation i.e. does the correlation appear at the perturbative or at the nonperturbative level in the generator?' For the perturbative level, there are two possibilities: matrix element and parton shower. A matrix element approach is limited to $O\left(\alpha_{s}^{2}\right)$ which automatically builds in a negative contribution to the correlation - if there are three partons on one side, $P_{t}$ on the other can only be zero. Generally therefore a parton shower treatment is preferred if this artificial bias is to be avoided. For the fragmentation stage the key requirement for a correlation to be induced is that the presence of a gluon on one side, say side 2, can result in hadrons on side 1 which carry some fraction of the gluon momentum ${ }^{1}$. Some ways in which this can happen will be examined.

\subsection{Independent fragmentation}

The simplest example of a fragmentation-induced correlation is furnished by an independent fragmentation model (IFM). The jet of particles that result from the fragmentation of a parton will have an angular spread around the parton momentum vector both from the intrinsic transverse momentum distribution given to the $q \bar{q}$ pairs that create the primary hadrons and from any subsequent hadron decays. The angular spread of the resulting jet may be sufficient for some particles which belong to the fragmentation of one parton to cross over to the other side. The probability of significant crossover, and the magnitude of the momentum transferred, will increase both with the energy of the radiated parton and its angle to the thrust axis, i.e. with $P_{t 2}$.

\footnotetext{
${ }^{1}$ If a small fraction $\alpha$ of a high $P_{t}$ system is carried over to the other side, this will contribute a positive term $\approx\left(\alpha P_{t}\right)\left((1-\alpha) P_{t}\right) \approx \alpha P_{t}^{2}$ to the correlation; the transfer of an isolated particle with $\delta P_{t}$ from one side to the other will contribute $-\left(\delta P_{t}\right)^{2}$, clearly negative.
} 
Tests were made using an IFM configuration of JETSET. The simulation was based on a matrix element with scale optimization of $\alpha_{s}$ and also with energy and momentum conservation. Parameters were tuned to ALEPH data. In spite of the negative bias from the limitation to $O\left(\alpha_{s}^{2}\right)$, the overall simulation gave a correlation at generator level of $+0.068 \pm 0.0015$, somewhat closer to the ALEPH measurement than JETSET parton shower (Table 2). Investigations showed that the rescaling of $\alpha_{s}$ was very important in reducing the negative impact of the matrix element and that both resonance decays and the jet intrinsic transverse momentum made important contributions at the fragmentation stage. There was a small but significant negative contribution attributed to the method used to force energy and momentum conservation.

The IFM leads to a certain pattern of particle and momentum flow between jets, the same flow as that which helps to produce the correlation. It is known that the model fails to describe well the detailed form of such flows and more generally gives a poor fit to data at LEP energies. Given also its theoretical weaknesses, it is not regarded as a good basis for an explanation but it does give a simple picture of how a correlation can occur.

\subsection{The JETSET explanation}

Unlike the IFM, the parton shower plus string JETSET, as described in section 3 , is known to give a good description of data over a wide range of phenomena, including interjet particle flow. Furthermore, investigation will show that the model is rather specific on the crucial effects that are found to lead to the correlation and gives considerable insight into the processes involved.

In this and the next section the analyses will concentrate on $d \bar{d}$ events only, with initial state radiation switched off. These studies are made at generator level, neglecting detector effects. To facilitate comparison between partons and hadrons, the same parton level thrust axis is used to partition both partons and hadrons. None of these simplifications has an important effect on the correlation.

Figure 2(a) shows $\overline{P_{t 1}}\left(P_{t 2}\right)$ for $d \bar{d}$ under the conditions described and presents two striking features. Firstly the rise in $\overline{P_{t}}$ below $P_{t 2}=10 \mathrm{GeV} / \mathrm{c}$ for the hadrons, whilst somewhat steeper, is almost as large as that seen with the full, JETSET reconstructed events (Fig. 1(d)). The correlation seen in the reconstructed events therefore still is present under these conditions; the value for the hadrons is $C=0.054 \pm 0.001$ at a $P_{t, \max }$ of $25 \mathrm{GeV} / \mathrm{c}$ and $0.055 \pm 0.001$ at a $P_{t, \max }$ of $10 \mathrm{GeV} / \mathrm{c}$. The effect appears strongly in the 'two jet' class of events with no flavour mixture present. Secondly there is a major clue as to origin in that almost no correlation is seen at the parton level: $C=0.002 \pm 0.001$. Although there are several processes that must at some level lead to a correlation in the parton cascade, such behaviour is not altogether unexpected in that, after the first bifurcations on each side, the two quark showers in JETSET start to evolve independently. The result offers strong evidence that the correlation in JETSET arises almost entirely at the non-perturbative, fragmentation stage.

The string itself will produce a coupling. Figure 3 shows a $d \bar{d} g$ event at a given time after formation. As the $d, \bar{d}$ and $g$ separate, colour forces lead to an expanding string, of tension $\kappa$, 
shown as a dashed line stretched from $d$ to $g$ to $\bar{d}$. The string break-up probability depends on elapsed proper time $\tau$ (and only $\tau$, if far from the ends) [10]. Any fragment of the string that finishes on side 1 will contain some of the gluon momentum, i.e. will introduce a $P_{t}$ coupling from side 2 to side 1 . The average magnitude of this coupling can be estimated in terms of $\kappa, \tau$ and the gluon energy and angle and compared with the observed average increase in $P_{t}$ during fragmentation. Good agreement is found in the variation of the increase over a range of energies and angles. In such a model, contributions from resonance decays and momentum fluctuations transverse to the string lead primarily to fluctuations around the momentum transferred by the string and therefore make only a small contribution to the average $P_{t}$.

Full parton showers, of course, are more complicated, with typically 6 partons and a string, or strings, that can cross more than once. Since the order of the partons along a string is given, adjacent partons which straddle the divide can be identified. Two cases are considered. The simpler is a generalization of Fig 3 in which there is a single $q$ on side 1 and possibly several gluons $g_{1} g_{2} \ldots$ on side 2 where, starting from the $q$, the suffix denotes the order along the string. In this case $\Delta P_{t 1}$, the average $P_{t}$ transfer to the $q$ expected from the string, can again be estimated directly in terms of the angle and energy of $g_{1}$. The actual $\overline{P_{t 1}}$ found for the hadrons at given $\Delta P_{t 1}$ is plotted against $\Delta P_{t 1}$ in Fig. 2(b). The straight line is drawn with unit gradient; over most of the range, and for most of the events, the estimate gives a good description of the coupling. More generally a string will couple $g_{i}$, at polar angle $\theta_{i}$, on side 1 with $g_{i+1}$, at polar angle $\theta_{i+1}$, on side 2 . In JETSET the azimuthal angle of gluon emission is randomly distributed around the direction of a radiating parton. Tests showed that, after azimuthal averaging of $g_{i}$, at low $\theta_{i}$ the coupling from $i+1$ to $i$ was still close to that expected with a single $q$ on side 1 . This was confirmed with the full JETSET events; as parton-level $P_{t 1}$ is raised, the change in average $P_{t 1}$ in passing from parton to hadron level shows a dependence on the predicted $\Delta P_{t 1}$ similar to that seen in Fig. 2(b) though with a slowly falling gradient. By combining unassociated half events and using the predicted $\Delta P_{t}$ transfers (modified by these gradients), the string couplings were shown to be adequate to explain the level of correlation seen in the JETSET data.

The 'JETSET picture' is therefore that the coupling and ensuing correlation arise primarily through non-perturbative colour forces between adjacent partons which transfer momentum between the two sides.

\subsection{The ARIADNE explanation}

The ARIADNE generator [11] uses the same string routine as JETSET but the parton cascade starts from the premise that since colour is a conserved quantity the colour dipole, rather than the parton, should be the independent radiator. A detailed comparison between ARIADNE at the two gluon level and second order perturbative $Q C D$ has been made [12]. It shows generally good agreement, when averaged over a set of events, with ARIADNE, including the minimum colour disturbance treatment $[11,12]$ of the dipole's recoil. This gives some indication that ARIADNE can be used to extrapolate beyond $O\left(\alpha_{s}^{2}\right)$.

ARIADNE 4.2 events were directly generated using tuned [8] parameters, again concentrating on $d \bar{d}$ as described above. The main result is shown in Fig. 2(c) and can be directly compared 
with the corresponding Fig. 2(a) for JETSET. The correlations are given in Table 4. The increase at the fragmentation stage, i.e. from parton to hadron, is similar in magnitude to that seen with JETSET; this, however, is true only for tuned values. The major difference is that the parton level itself shows a substantial correlation, with the ubiquitous rise in $\overline{P_{t 1}}\left(P_{t 2}\right)$ with increasing $P_{t 2}$, followed by a near plateau above about $11 \mathrm{GeV} / \mathrm{c}$. There exists therefore the basis for a perturbative QCD explanation of the correlation.

\begin{tabular}{|c|c|c|}
\hline & $C_{\text {parton }}$ & $C_{\text {hadron }}$ \\
\hline JETSET & $\mathbf{0 . 0 0 2} \pm \mathbf{0 . 0 0 1}$ & $\mathbf{0 . 0 5 4} \pm \mathbf{0 . 0 0 1}$ \\
ARIADNE & $\mathbf{0 . 0 6 2} \pm \mathbf{0 . 0 0 1}$ & $\mathbf{0 . 1 1 6} \pm \mathbf{0 . 0 0 1}$ \\
\hline
\end{tabular}

Table 4. Correlations for JETSET and ARIADNE $d \bar{d}$ partons and hadrons $\left(P_{t, \max }=25 \mathrm{GeV} / \mathrm{c}\right)$.

Considering a $q \bar{q}$ dipole 1-2 (Fig. 4), formed of partons 1 and 2, the radiation of a gluon 3 from such a dipole is described in terms of a quantity $p_{\perp}$ where, for massless quarks,

$$
p_{\perp}^{2}=\frac{S_{23} S_{31}}{S_{12}}
$$

$S_{i j}$ being the invariant mass squared of dipole $i j$. The original 1-2 is replaced by 1-3 and 3-2. If one dipole radiates a further gluon, parton 4 , ordering in $p_{\perp}$ leads to a preference for this gluon to be radiated by 1-3 rather than 3-2. The centre of mass velocity of 1-3 (opposite parton 2) will boost 4 and hence tend to transfer momentum from side 2 to side 1 . The other feature of particular importance in the model is that, unlike JETSET, the entire event is considered at each branching, giving a correlation which can increase significantly with the number of branchings.

The ARIADNE hadron correlation of 0.116 for $d \bar{d}$ is significantly higher than the value of 0.084 measured in the data. (Table 2). To investigate this in more detail ARIADNE events were also generated with the full range of flavours, initial state radiation and the thrust axis pertaining to the generator level hadrons. The correlation was found to be $0.126 \pm .001$, little different from the $d \bar{d}$ above. After correcting for detector effects (section 3.2), this became $0.115 \pm 0.005$. A systematic error in the correction of half its value has been assumed. With the tuned parameters, ARIADNE clearly predicts too high a correlation between the hadrons.

At the parton level, apart from the assignment of quark masses, ARIADNE has just two parameters: $\Lambda_{Q C D}$ and the cut-off $p_{\perp, \text { min }}$, with ALEPH values 0.225 and $0.73 \mathrm{GeV}$ respectively. The value for $p_{\perp, \min }$ will depend also on the string parameters chosen to give a good description of the final hadrons and must have a certain degree of arbitrariness. Pursuing the perturbative approach as described by ARIADNE, and invoking Local Parton Hadron Duality (LPHD) [13] as justification for considering the partons alone, it is interesting to allow the evolution to proceed further. The effect of varying $p_{\perp, \text { min }}$ is shown in Fig. 2(d), with more details in the Table 5 . 


\begin{tabular}{|c|c|c|c|c|}
\hline$p_{\perp, \min }$ & $\bar{n}_{\text {parton }}$ & $\overline{P_{t}}$ & $\sigma$ & $C_{\text {parton }}$ \\
\hline 0.25 & 21.5 & 7.88 & 5.56 & 0.085 \\
0.30 & 16.3 & 7.84 & 5.59 & 0.078 \\
0.40 & 12.0 & 7.78 & 5.66 & 0.071 \\
0.50 & 9.9 & 7.71 & 5.70 & 0.067 \\
0.75 & 7.4 & 7.55 & 5.87 & 0.059 \\
1.00 & 6.0 & 7.35 & 6.04 & 0.051 \\
2.00 & 4.1 & 6.52 & 6.59 & 0.045 \\
5.00 & 2.7 & 4.34 & 7.04 & 0.029 \\
\hline
\end{tabular}

Table 5 . The behaviour of ARIADNE partons $(d \bar{d})$ as a function of the cut-off $p_{\perp, \min }$. The statistical error on $C$ is $\pm \mathbf{0 . 0 0 1 5}$.

The correlation does indeed increase steadily as $p_{\perp, \min }$ is reduced and it is even possible to get quite good numerical agreement at the parton level with ALEPH data. Such a result, of course, can only be indicative of the possible consequences of a full, perturbative approach. The other noteworthy feature is that a significant part of the correlation is already present at $p_{\perp, \min }$ $=5 \mathrm{GeV} / \mathrm{c}$, i.e. early in the event when the total average number of partons present is only 2.7 - less than an average of one radiated gluon - and also indicates that a proper, perturbative approach would be expected to give a positive contribution to the correlation in this region.

\section{Cluster correlations}

In JETSET hadrons and ARIADNE partons there is the basis for two apparently very different kinds of explanation for the correlation. JETSET exemplifies a non-perturbative origin; the main correlation is produced after the end of the parton shower as the hadrons are formed. In the $d \bar{d} g$ event of section 4.2 , if the string fragments between the $d$ and the $g$ are shared equally, each parton acquires particles with a total momentum transverse to itself of the order of $\kappa \tau \sin \left(\theta_{g} / 2\right)$, typically $\approx 1 \mathrm{GeV} / \mathrm{c}$, a figure also typical of other non-perturbative effects such as momentum transverse to the string, or transverse to the parton in IF, or hadron decays. This can be compared with the much larger $p_{\perp}$ values in the perturbative phase of the event evolution at which a significant correlation is formed with ARIADNE partons; $p_{\perp}$ is also closely related to a transverse momentum. Could the difference in scale be used to distinguish perturbative and non-perturbative contributions to the correlation?

The Durham jet cluster algorithm [14] is used to define a quantity directly sensitive to the transverse momentum scale. The controlling parameter in the algorithm is $y_{c u t}, y_{i j}<y_{\text {cut }}$, where $y_{i j}$ between two particles $i$ and $j$ is defined as

$$
y_{i j}=\frac{2 \min \left\{E_{i}^{2}, E_{j}^{2}\right\}\left(1-\cos \theta_{i j}\right)}{E_{v i s}^{2}}
$$

where $E_{i}$ and $E_{j}$ are the respective energies of, and $\theta_{i j}$ the angle between, the two particles. $E_{v i s}$ is replaced by $\sqrt{s}$, the known total energy. The algorithm recombines $j$, say a string fragment, 
with $i$ provided that $\sqrt{y_{c u t} s}>2 E_{j} \sin \left(\theta_{i j} / 2\right)$. The four-momentum of the resulting cluster is taken to be the sum of the four-momenta of $i$ and $j$ (the ' $\mathrm{E}$ ' scheme). The quantity $\sqrt{y_{\text {cut }} s}$ is written as $m_{y} ; m_{y}$ itself is closely related to the transverse momentum of $j$ with respect to $i$. The correlation is examined as a function of $m_{y}$; at an appropriate value of $m_{y}$ one could effectively be studying the correlation at the early parton stage where the difference in the two models should be greatest.

The detailed procedure is as follows. Each event is analysed as before. The thrust axis and $P_{t}$ values are determined and events with either $P_{t}>25 \mathrm{GeV} / \mathrm{c}$ are rejected. The cluster algorithm is applied at 12 increasing values of $m_{y}$ ranging from 0 to $10 \mathrm{GeV}$. The first value, $m_{y}=0$, corresponds to the analysis with hadrons. At each stage the event is partitioned with respect to the original thrust axis and $P_{t}$ determined from the cluster momenta for each half separately. This results in all in 12 pairs of $P_{t}$ values and finally 12 values of $C\left(m_{y}\right)$ from $m_{y}=$ 0 to $10 \mathrm{GeV}$.

The method was tested, and a suitable range of $m_{y}$ identified, by examining the light quark events. For JETSET this was most conveniently done by extracting the $u$ and $d$ flavour events from the ALEPH Monte Carlo data set. Figure 5(a) shows the correlation coefficient as a function of $m_{y}$. Looking first at the partons, the flat region at low $m_{y}$ is an artefact of the $m_{\min }$ parameter $(1.9 \mathrm{GeV})$ which controls the virtuality cut-off and hence terminates the parton shower. As $m_{y}$ increases, $C$ becomes negative. This is attributed to an effect of the algorithm which can transfer particles, and hence $P_{t}$, from one side to the other, giving a negative contribution to the correlation even when none was previously there. The key point however is that the effect of the string-induced correlation is largely confined to $m_{y}<2 \mathrm{GeV}$ and that above this, apart from a small offset, the hadron curve does follow the partons.

Similar behaviour can be seen for $d \bar{d}$ ARIADNE, Fig. 5(b). Again the difference between hadrons and partons is largely confined to $m_{y}<2 \mathrm{GeV}$. On the other hand, pre-clustering at an $m_{y}$ value of $6 \mathrm{GeV}$ for example and then forming the correlation does show a large difference between the two models.

Figure 5(c) compares ALEPH data with the full JETSET reconstructed events. As already seen in Table 2, JETSET is lower than the data at $m_{y}=0$; in fact it remains significantly below the data throughout the range of $m_{y}$. The figure also includes the JETSET events at generator level. Over most of the range, detector effects lead to a reduction in the correlation of about 0.003 , clearly small on the scale of the differences observed.

The full ARIADNE hadron simulation (all flavours) is compared with the data in Fig. 5(d). The ARIADNE events are uncorrected for detector effects but the difference between generated and reconstructed is expected to be small and fairly constant. The full line shows the behaviour predicted by the pure parton model in which the cascade cut-off is reduced to $p_{\perp, \min }=0.30$ $\mathrm{GeV} / \mathrm{c}$, emphasizing that under these circumstances the partons alone can contain the principal features and magnitude of the correlation: a rapid variation in what would otherwise be called the non-perturbative region plus a significant deeper parton structure.

The difference $\Delta C\left(m_{y}\right)=C_{M C}\left(m_{y}\right)-C_{A L E P H}\left(m_{y}\right)$ between ALEPH data and Monte 
Carlo is examined in Fig. 6 for three of the simulations shown in Fig. 5. The result for JETSET shows that there is no evidence from $\Delta C_{J E T S E T}$ of any significant structure at low $m_{y}$. Although JETSET underestimates the value of $C$ it describes well the variation of $C$ with $m_{y}$ in the string region below $2 \mathrm{GeV}$. This is a clear indication of the importance of non-perturbative QCD effects in the formation of the observed correlation. The difference that there is persists over the entire $m_{y}$ range studied, up to $10 \mathrm{GeV}$, pointing to a lack of correlation at the hard parton level early in the evolution of the shower and therefore for the need for an important perturbative contribution to the correlation.

The high level of correlation predicted for ARIADNE hadrons persists throughout the $m_{y}$ range, again without any significant structure in the string region. There remains a large excess correlation which falls slowly with increasing $m_{y}$, this time indicating too strong a correlation at the hard parton level.

Though it still has a discrepancy at high $m_{y}$, the pure parton cascade generated by ARIADNE with a $p_{\perp, \text { min }}$ cut-off of $0.3 \mathrm{GeV} / \mathrm{c}$ - a model one might refer to as 'naive LPHD' is, if anything, the closest of the three to describing the data but evidently lacks some of the correlation introduced in JETSET by a string.

\section{Conclusions}

A significant, positive, transverse momentum correlation which cannot be explained by energymomentum conservation, flavour conservation, the imposition of an event axis or imperfect event reconstruction, exists between the two sides of hadron events.

A similar type of correlation is found in reconstructed JETSET events. For the chosen data set with a $P_{t, \max }=25 \mathrm{GeV} / \mathrm{c}$, the correlation has a magnitude of about $2 / 3$ of that seen in the ALEPH data. Within the context of the model, the correlation is shown to be largely attributable to momentum coupling via the non-perturbative Lund 'string'; the correlation develops in the final stages of an event as the coloured partons separate. An alternative description based on independent fragmentation, in which the hadrons from a parton jet on one side cross over to the other, can also generate the correlation.

Studies with the ARIADNE generator, in which the parton shower develops as a whole, indicate an important contribution to the correlation at the parton, or perturbative level. The hadron correlation predicted by ARIADNE is too high, about $30 \%$ above the measured value. The parton correlation is such that by allowing the parton cascade to develop further, it is possible to produce, at the parton level alone, a value close to the required magnitude i.e. without any string or other non-perturbative effect.

A technique in which the correlation is examined as a function of a jet-clustering parameter $m_{y}=\sqrt{y_{\text {cut }} s}$ is introduced and is applied to the three models: JETSET hadrons; ARIADNE hadrons and the ARIADNE parton cascade. The results give further insight into the strengths 
and failures of the models in describing the data. It is concluded that both non-perturbative and perturbative effects make important contributions to the observed correlation.

\section{Acknowledgements}

We are grateful to B. Andersson for a helpful discussion on ARIADNE. We thank our colleagues from the accelerator divisions for the successful operation of LEP, also the engineers and technicians from our institutions for their contributions to ALEPH. Those of us from nonmember countries thank CERN for its hospitality.

\section{References}

[1] P.Abreu et al., DELPHI collab., Z. Phys. C 50 (1991) 185

[2] P.Abreu et al., DELPHI collab., Phys. Lett. B 276 (1992) 254

[3] R.Akers et al., OPAL collab., Phys. Lett. B 320 (1994) 417

[4] D.Decamp et al., ALEPH collab., Nucl.Instr. Meth. A 294 (1990) 121

[5] D.Buskulic et al., ALEPH collab., Nucl. Inst. Meth. A 360 (1995) 481

[6] T.Sjöstrand and M.Bengston, Comp. Phys. Comm. 43 (1987) 367

[7] M.Bengston and T.Sjöstrand, Phys. Lett. B 185 (1987) 435

[8] D.Buskulic et al., ALEPH collab., Z. Phys. C 55 (1992) 209

[9] D.Buskulic et al., ALEPH collab., Phys. Lett. B 313 (1994) 441

[10] B.Andersson, G.Gustafson and B.Söderberg, Z. Phys. C 20 (1983) 317

[11] L.Lönnblad, Comp. Phys. Comm. 71 (1992) 15

[12] B.Andersson, G.Gustafson and C.Sjögren, Nucl. Phys. B 380 (1992) 391

[13] Ya.I.Azimov et al., Phys.Lett. B 165 (1985) 147; Z. Phys. C 27 (1985) 65

[14] W.J.Stirling, J. Phys. G 17 (1991) 1567 

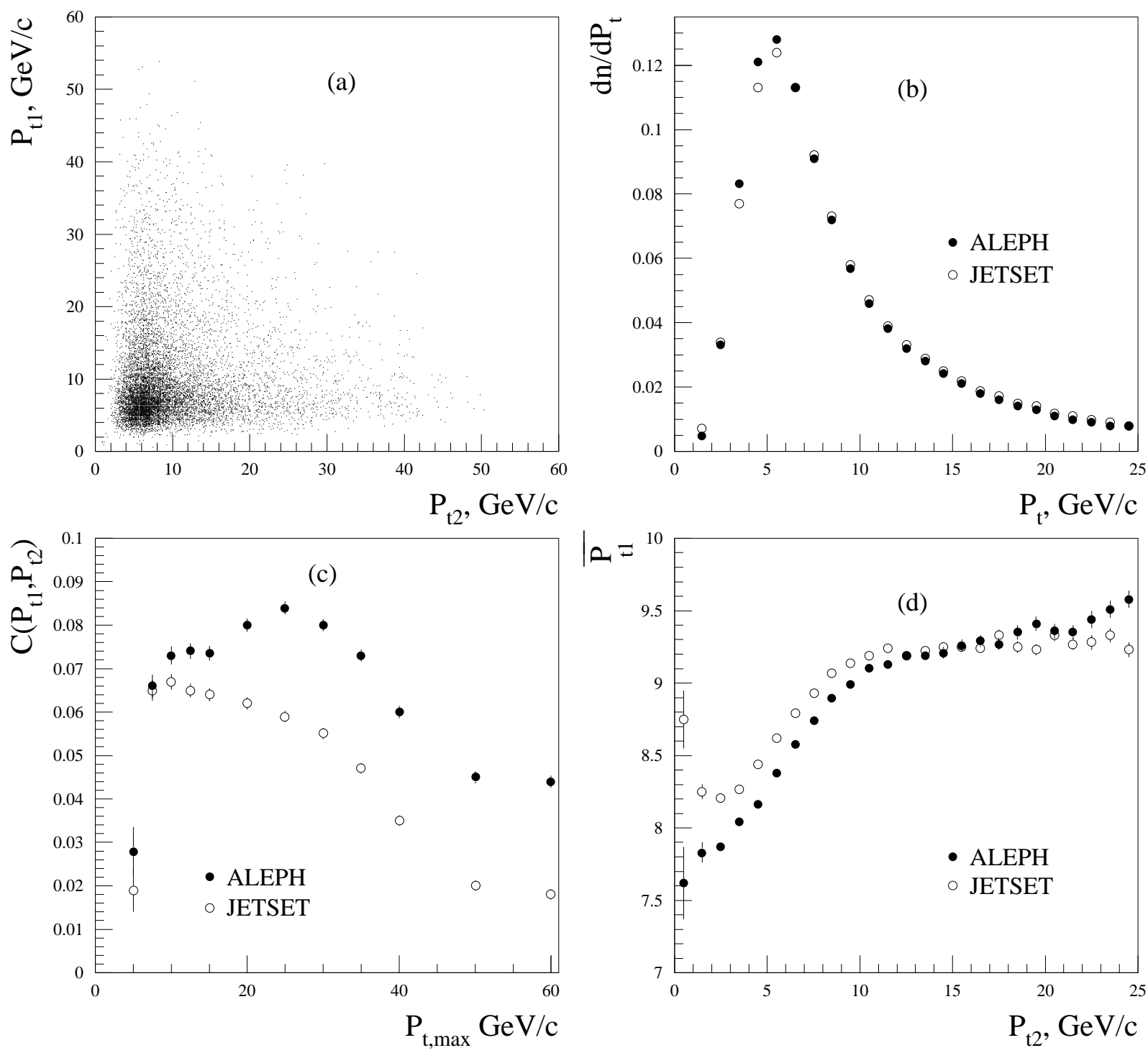

Figure 1: 1992 ALEPH data and reconstructed JETSET events. a) Scatter plot of $P_{t 1}$ versus $P_{t 2}$ for ALEPH data. b) $P_{t}$ distribution for events with $P_{t 1}, P_{t 2}<25 \mathrm{GeV} / \mathrm{c}$. c) Correlation as a function of $P_{t, \max }$ when only events with $P_{t 1}, P_{t 2}<P_{t, \text { max }}$ are accepted. d) Average $P_{t 1}$ as a function of $P_{t 2}$ for the events in (b). 

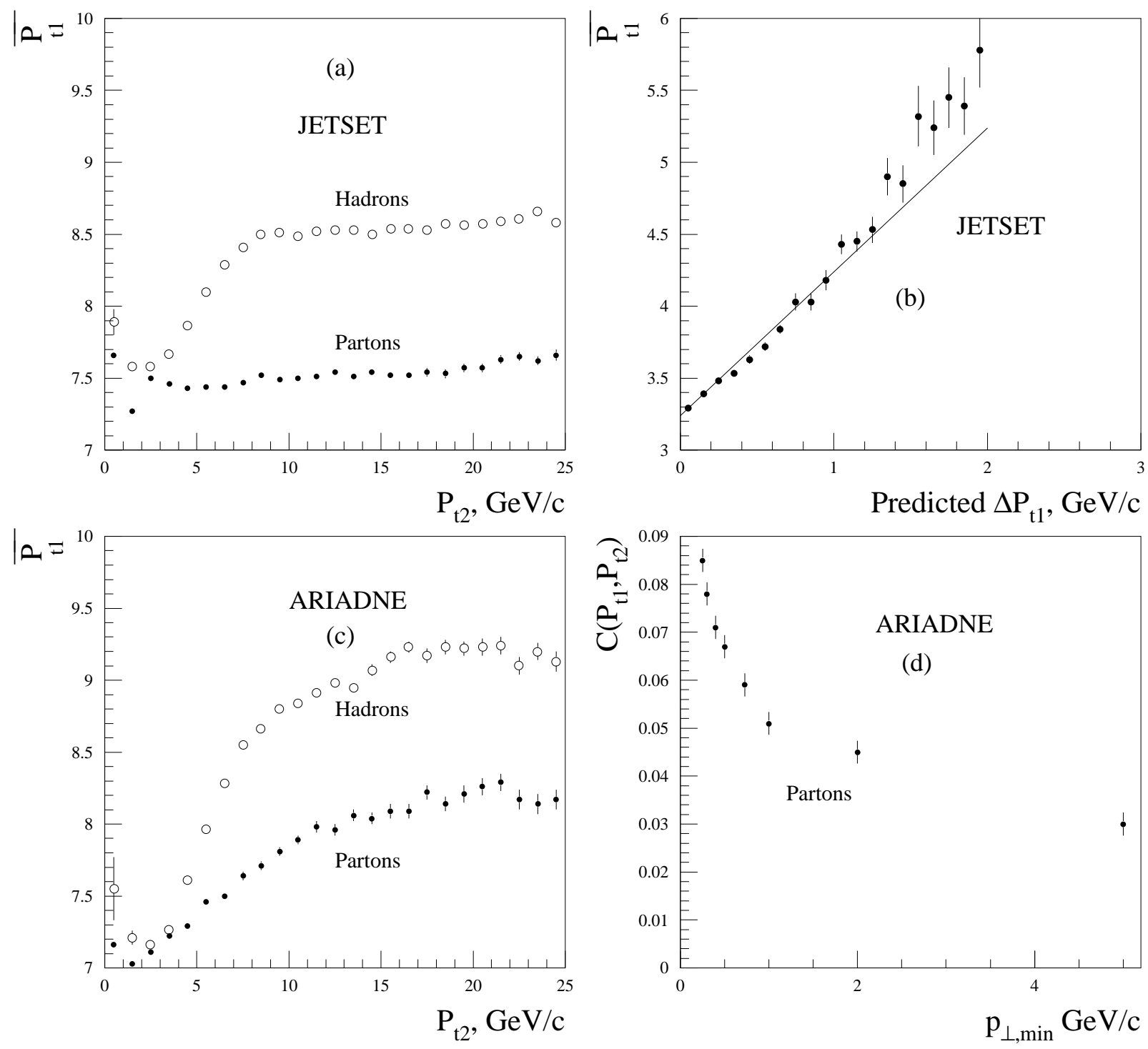

Figure 2: a) Average $P_{t 1}$ as a function of $P_{t 2}$ for pure $d \bar{d}$ partons and hadrons in JETSET. b) Evidence for the origin of the correlation in JETSET. c) As in a) but for ARIADNE. d) ARIADNE parton correlation as a function of $p_{\perp, \min }$. 


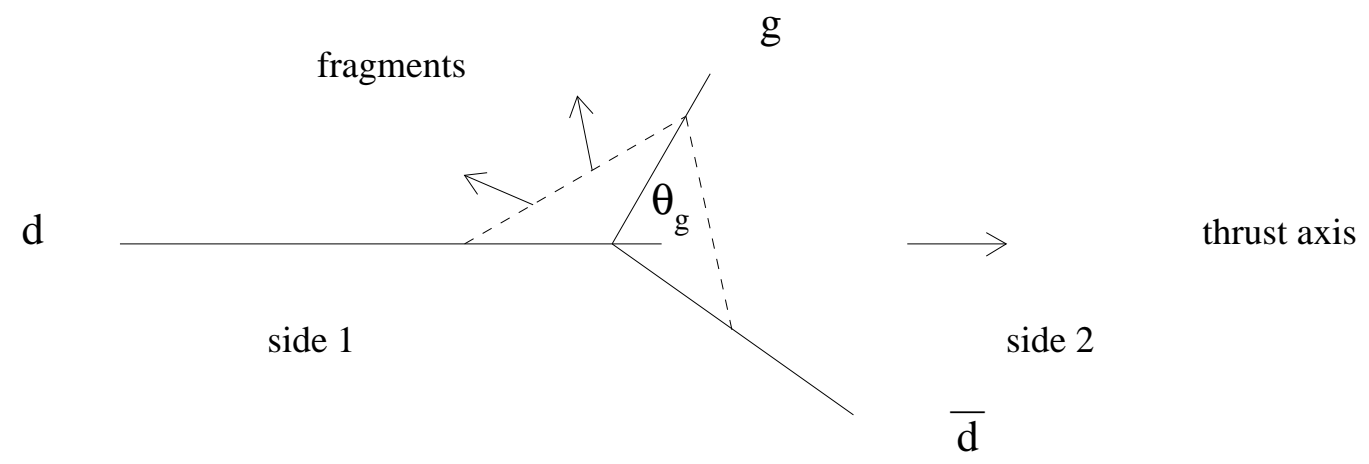

Figure 3: Diagram of a $d \bar{d} g$ event. The dashed line represents the string.

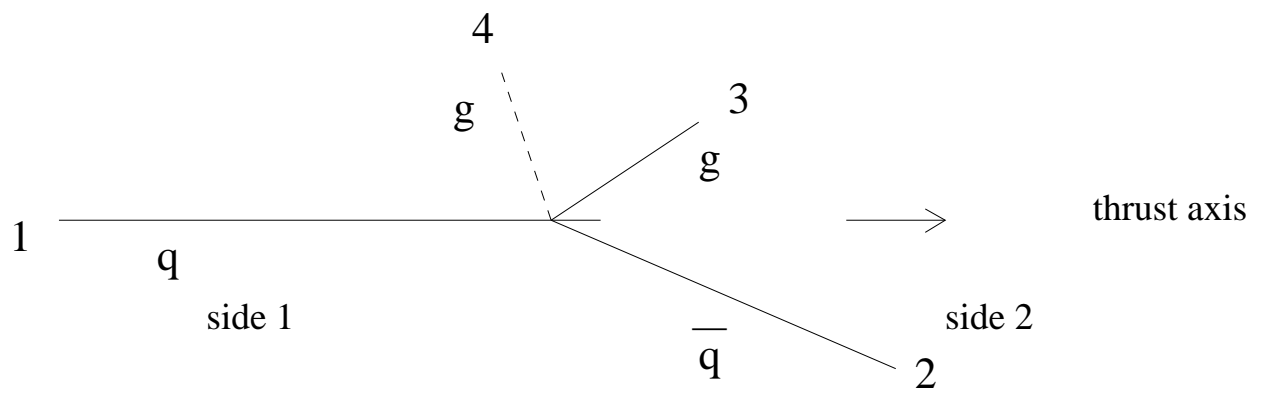

Figure 4: Dipoles in ARIADNE. The dashed line represents the radiation of a second gluon. 

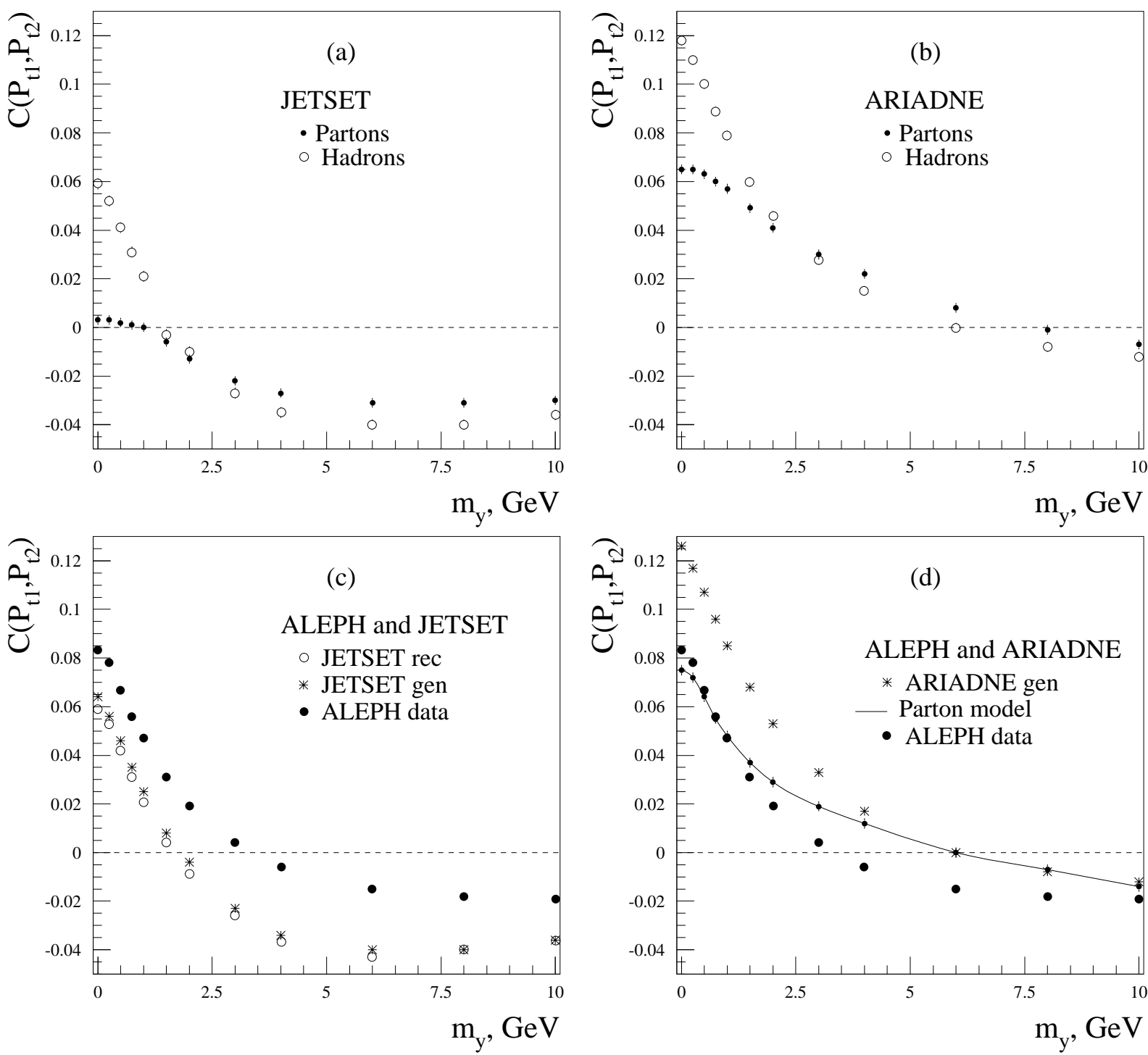

Figure 5: Application of the Durham jet cluster algorithm to JETSET, ARIADNE and ALEPH data showing how the $P_{t}$ correlation $\left(P_{t, \max }=25 \mathrm{GeV} / \mathrm{c}\right)$ varies with $m_{y}=\sqrt{s . y_{\text {cut }}}$. a) JETSET $u \bar{u}+d \bar{d}$ partons and generator-level hadrons. b) Similar to a) for ARIADNE. c) Comparison of ALEPH data and the full reconstructed JETSET events (all flavours), also the corresponding generator level. d) ALEPH data and generator level ARIADNE hadrons (all flavours), also a pure parton model with $p_{\perp, \text { min }}=0.3 \mathrm{GeV}$ (full line). 


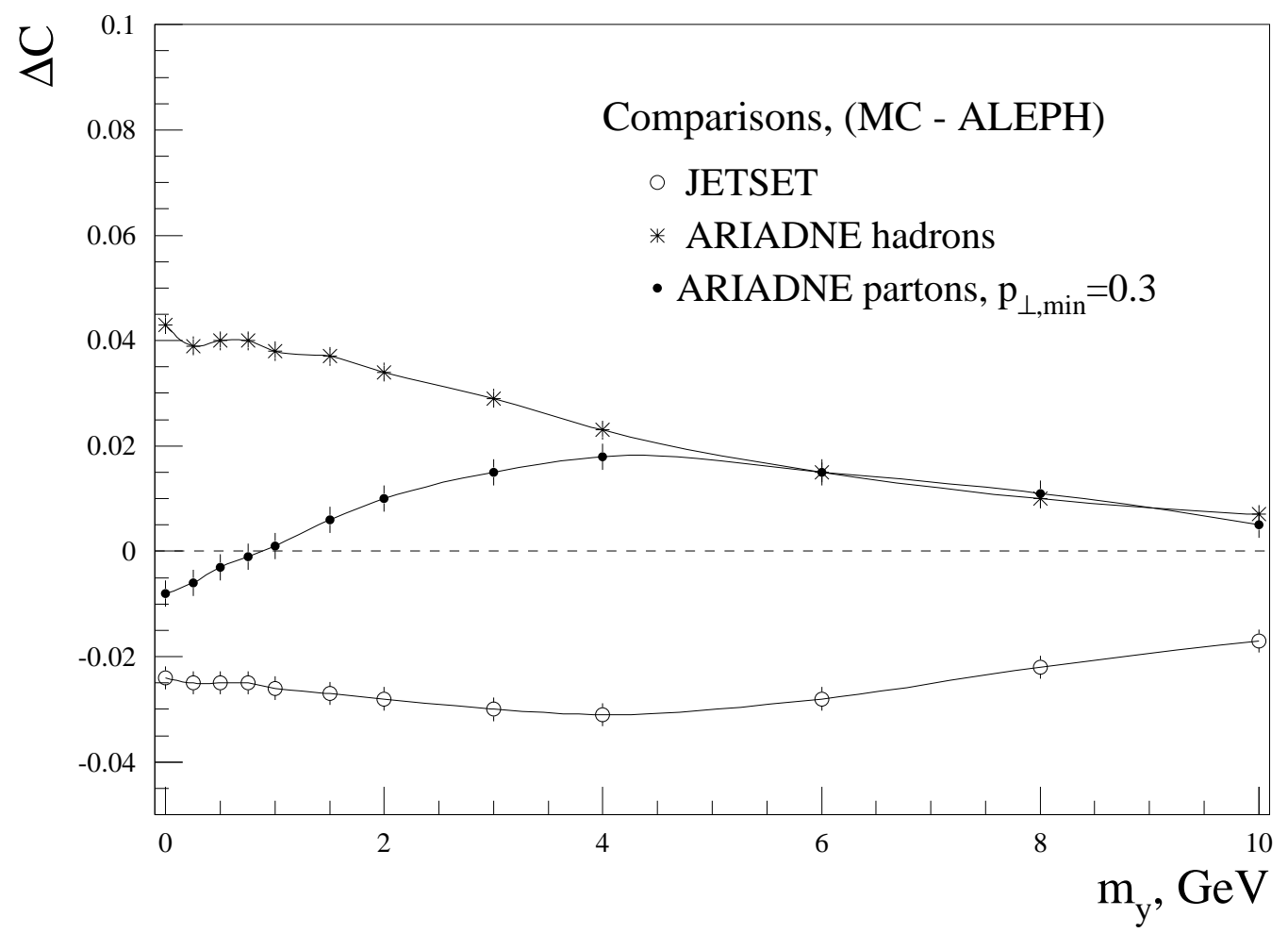

Figure 6: The correlation difference $\Delta C=C_{M C}-C_{A L E P H}$ for JETSET reconstructed events, ARIADNE hadrons and ARIADNE partons with $p_{\perp, \text { min }}=0.30 \mathrm{GeV}$ 